\title{
ROBOT TRANSPORTADOR Y DISTRIBUIDOR DE OBJETOS SEGÚN SU PESO
}

\section{CARRIER AND OBJECTS DISTRIBUTOR ROBOT AC- CORDING TO OBJECT'S WEIGHT}

\section{RESUMEN}

El presente artículo presenta los criterios de diseño para la construcción de un robot omnidireccional, a partir del planteamiento de modelos matemáticos, como el modelo cinemático directo e inverso, con el cual es posible interpretar que tipo de desplazamiento posee el robot y en qué circunstancias se da dicho desplazamiento; el estudio de la resistencia de materiales permite conocer si el material con el cual se pretende construir la estructura es lo suficientemente resistente para soportar no solo su propio peso, si no también el peso del objeto que debe transportar. Finalmente se establece la fuerza que deben ejercer los actuadores para vencer la fuerza de fricción. Así como el esquema de control general del proyecto y de los motores.

Palabras clave: fuerza de fricción, fuerza normal, modelos cinemáticos, resistencia de los materiales.

\begin{abstract}
This paper shows the design criteria to build an omnidirectional robot from mathematical modeling approaches, such as: the direct and inverse kinematic model, which allows to interpret which kind of movement has the robot performed and under what kind of circumstances, the study of the material resistance will allow us to know how resistant should it be to bear not only its own weight but also the object to be transported. On the other hand the strength that must be performed by actuators to overcome the friction power. As these parameters will enable the robot move. Well as the control scheme of the project and the engines. It will also embrace the project general control and engines schema.
\end{abstract}

Key words: frictional force, kinematic models, material strength, normal force.

\section{Luis Efrén Barrero Páez}

Magister en Ingeniería Electrónica y de Computadores

Universidad de San Buenaventura

luisbarreropaez@gmail.com

Bogotá, Colombia

\section{Andrés Felipe Villegas Caballero}

Ingeniero Electrónico

Universidad Manuela Beltrán

felipevillegas81@gmail.com

Bogotá, Colombia

Diana Carolina Gómez Gómez

Ingeniero Electrónico

Universidad Manuela Beltrán

carola369@gmail.com

Bogotá, Colombia

Tipo: Artículo de investigación

Fecha de Recepción: Octubre 1 de 2013

Fecha de Aceptación: Diciembre 22 de 2013 


\section{INTRODUCCIÓN}

A través de la historia, el hombre gracias a su curiosidad e ingenio ha dado lugar a grandes inventos que han hecho de nuestra vida algo más sencillo. Como la mayoría de máquinas creadas por el hombre buscan mejorar nuestro estilo de vida y a partir de la revolución industrial hacer que la industria sea más productiva por medio de la automatización, lo cual hace parte del enfoque la robótica [1]. Es por eso que en la actualidad, los robots se han convertido en una excelente opción para generar producción en masa y automatizar procesos industriales, así como facilitar ciertas actividades humanas que suelen ser repetitivas [2] o de alto riesgo. Además, debido a la rapidez y precisión con la que ejecutan las tareas para las que fueron creados, incrementan la eficiencia y productividad de la compañía que los implemente.

Por lo tanto este artículo tiene como propósito mostrar el diseño de un robot omnidireccional haciendo uso de modelos matemáticos tales como los modelos cinemático inverso y directo, la segunda ley de Newton y el estudio de la resistencia del material, con el fin de que sea utilizado como base para el desarrollo de nuevas tecnologías que involucren la clasificación de objetos y el transporte de los mismos, un ejemplo de ello son las empresas que manejan encomiendas, pues dichas empresas cobran el valor de la encomienda según el peso de la misma.

Actualmente existen robots como el patentado por Porsche, utilizado para llevar objetos muy pesados como motores de automóviles, los robots creados por Kiva Systems para la distribución de mercancía [3] o el Round a Bot, es capaz de cargar hasta 200kg [4]. Sin embargo existen otras posibles aplicaciones para los robots con este tipo de configuración incluyen desde la impresión de grandes superficies, guiado de herramientas de corte para perfiles de gran tamaño hasta vehículos teledirigidos de inspección, investigación u ocio [5].

\section{MODELO CINEMÁTICO}

Un robot omnidireccional se caracteriza por- que puede moverse en cualquier dirección [6] sin necesidad de reorientación, debido a que utiliza ruedas omnidireccionales, las cuales constan de una rueda normal con rodillos ubicados de forma perpendicular a la dirección normal de la rueda. Entonces cuando se aplica una fuerza lateral, el robot se desplaza sobre los rodillos, lo que permite que haya una componente de velocidad en el eje $\mathrm{x}$.

El modelo cinemático de este tipo de robot se define a partir de un triángulo equilátero en el cual se ubica una rueda en cada uno de sus vértices y por consiguiente cada rueda posee entre si un ángulo de separación de 120 grados como se muestra en la figura 1. Ahora la distancia del centro de las ruedas al centro del triángulo se denota como L, la velocidad angular de cada rueda se representa como w1, w2, w3 y por ultimo esta $r$ que hace referencia al radio de las ruedas].

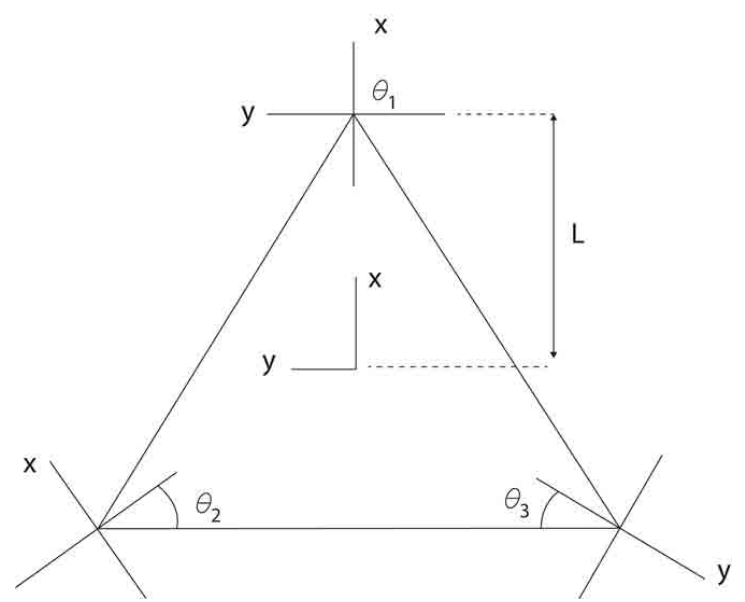

Figura 1. Esquema de la posición de las ruedas en un sistema de locomoción omnidireccional con tres ruedas

Donde los ángulos $\theta_{1}, \theta_{2}$ y $\theta_{3}$ toman los siguientes valores de acuerdo a su ubicación en el triángulo equilátero:

$$
\begin{aligned}
& \theta_{1}=0^{\circ} ; \theta_{2}=30^{\circ} ; \theta_{3}=150^{\circ} \\
& \mathrm{L}=0,1748[\mathrm{~m}] ; \mathrm{r}=0,12[\mathrm{~m}]
\end{aligned}
$$

Dicho modelo cinemático se presenta de dos formas: directa e inversa. De la primera se obtiene el desplazamiento y posición del robot 
con respecto a los ejes $\mathrm{x}, \mathrm{y}, \mathrm{z}$, a partir de la velocidad angular de las ruedas. Mientras que de la segunda se obtiene la velocidad angular de las ruedas a partir de la posición del robot y de su desplazamiento con respecto a los ejes de coordenadas. Sin embargo antes de realizar el análisis es necesario fijar unos parámetros, como la dirección de giro de las ruedas cuando se desplaza hacia delante, teniendo en cuenta su disposición. En este caso para las Ruedas 1 y 2 , el giro es positivo cuando se realiza en contra de las manecillas del reloj, mientras que la Rueda 3 cuando giran en el sentido contrario a las manecillas del reloj, consideramos el giro con signo negativo y viceversa, como se muestra en la figura 2 .



Figura 2. Convención de signos respecto a la dirección de giro de cada rueda cuando el robot se desplaza hacia delante

Por otro lado en el caso en que el robot gira sobre su propio eje en el sentido de las manecillas del reloj se considerara como un movimiento con signo negativo, y si se presenta el caso contrario, entonces será un movimiento con signo positivo.

A continuación se encuentran las matrices y ecuaciones que describen el modelo cinemático directo e inverso de un robot omnidireccional.

\subsection{Modelo cinemático directo}

A partir del modelo cinemático directo es posi- ble conocer cuánto debe avanzar, sobre que eje de coordenadas y en qué sentido gira cada una de las ruedas con respecto a las otras.

Para este cálculo se tiene el vector de posición $A p$ y el vector de velocidad angular $\varphi$ [6], los cuales aplican para el modelo directo e inverso y están dados por la ecuación (1) y ecuación (2).

$$
\begin{aligned}
& A p=\left[\begin{array}{l}
x \\
y \\
z
\end{array}\right] \\
& \varphi=\left[\begin{array}{l}
\omega_{1} \\
\omega_{2} \\
\omega_{3}
\end{array}\right]
\end{aligned}
$$

Para hallar el vector posición se utiliza la ecuación (3).

$$
\left[\begin{array}{l}
x \\
y \\
z
\end{array}\right]=\frac{r}{3} \cdot\left[\begin{array}{ccc}
\sin \theta_{1} & 2 \cos \theta_{2} & -2 \cos \theta_{3} \\
-2 \cos \theta_{1} & 2 \sin \theta_{2} & 2 \sin \theta_{3} \\
-1 / L & -1 / L & -1 / L
\end{array}\right]\left[\begin{array}{c}
\omega_{1} \\
\omega_{2} \\
\omega_{3}
\end{array}\right]
$$

A partir de la ecuación (3) se deducen las ecuaciones (4), (5) y (6) que permiten hallar las coordenadas y el desplazamiento en $\mathrm{x}, \mathrm{y}$, z.

$$
x=\left(\omega_{1} \cdot \sin \theta_{1}+\omega_{2} \cdot 2 \cos \theta_{2}-\omega_{3} \cdot 2 \cos \theta_{3}\right) \cdot \frac{r}{3}
$$

$$
\begin{gathered}
y=\left(-\omega_{1} \cdot 2 \cos \theta_{1}+\omega_{2} \cdot 2 \sin \theta_{2}+\omega_{3} \cdot 2 \cos \theta_{3}\right) \cdot \frac{r}{3} \\
z=\left(-\omega_{1} \cdot 1 / L-\omega_{2} \cdot 1 / L-\omega_{3} \cdot 1 / L\right) \cdot \frac{r}{3}
\end{gathered}
$$

Dichos valores se obtiene remplazando el ángulo correspondiente a la inclinación de cada una de las ruedas respecto al eje de coordenadas.

Por lo tanto para hallar el desplazamiento de cada una de las ruedas se remplaza en las ecuaciones (4), (5), (6) cada uno de los resultados obtenidos en la matriz según corresponda y el 
valor de L, la cual depende del tamaño del robot.

En este orden de ideas, la ecuación (7) representa el modelo cinemático directo, de la cual se concluye que el desplazamiento de la Rueda1 se rige por los valores que se encuentra en la primera columna de izquierda a derecha de la matriz $3 \times 3$, la Rueda 2 se rige por la segunda columna y la Rueda3 por la tercera. Además, también muestra que el valor que toma x corresponde a la primera fila de arriba hacia abajo, y a la segunda fila y $\mathrm{z}$ a la tercera fila como se observa en las ecuaciones. Por lo tanto para realizar el análisis del desplazamiento se asignan valores a las variables correspondientes a la velocidad angular de cada una de las ruedas, es decir a $\omega_{1}, \omega_{2}, \omega_{3}$.

$$
\left[\begin{array}{l}
x \\
y \\
z
\end{array}\right]=\frac{r}{3}\left[\begin{array}{ccc}
0 & \sqrt{3} & -\sqrt{3} \\
-2 & 1 & 1 \\
-1 / L & -1 / L & -1 / L
\end{array}\right]\left[\begin{array}{l}
\omega_{1} \\
\omega_{2} \\
\omega_{3}
\end{array}\right]
$$

Inicialmente se determina el desplazamiento de las ruedas cuando el robot rota sobre si mismo, asignando los siguientes valores a las variables de velocidad angular.

$$
\begin{aligned}
& \omega_{1}=1 \\
& \omega_{2}=1 \\
& \omega_{3}=1
\end{aligned}
$$

Se reemplazan los valores de $\omega$ en las ecuaciones (4), (5), (6), y se obtiene los siguiente resultados.

$$
\begin{aligned}
\mathrm{x} & =0[\mathrm{~m}] \\
\mathrm{y} & =0[\mathrm{~m}] \\
\mathrm{z} & =-0,68[\mathrm{~m}]
\end{aligned}
$$

Como se observa en los resultados, si reemplazamos la velocidad angular para cada rueda con una unidad positiva se obtiene solo el resultado de la componente en $\mathrm{z}$, la cual corresponde a la rotación del robot, con signo negativo, lo que significa que es robot está girando hacia la derecha, es decir en el sentido de las manecillas del reloj, como muestra la figura 3.

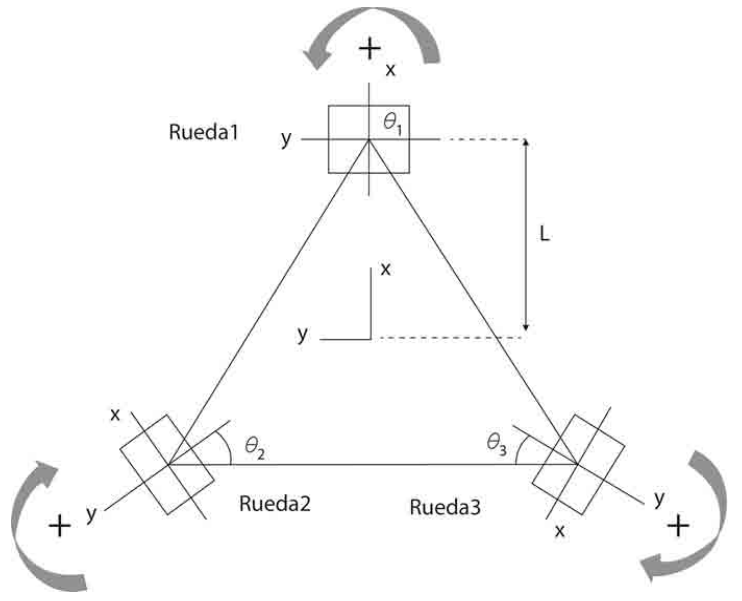

Figura 3. Rotación del robot en sentido horario

Si se desea conocer el desplazamiento del robot hacia adelante solo se reemplaza el valor de $\omega_{2}$ y $\omega_{3}$, que corresponden a la Rueda 2 y 3 respectivamente e incluimos el sentido de la rotación de cada rueda por medio del signo. La Rueda1 se le asigna el valor de cero debido a que se desplazara sobre los rodillos que posee, es decir que será "arrastrada" por el movimiento de los motores conectados a las Ruedas 2 y 3.

$$
\begin{array}{l|l|l}
\omega_{1}=0 & \mathrm{x}=0,14[\mathrm{~m}] \\
\omega_{2}=1 & \mathrm{y}=0[\mathrm{~m}] \\
\omega_{3}=-1 & \mathrm{z}=0[\mathrm{~m}]
\end{array}
$$

Entonces se observa en los resultados que el robot se esta desplazando solo sobre el eje $\mathrm{x}$ $0,14 \mathrm{~m}$, es decir alrededor de $14 \mathrm{~cm}$ como se muestra en la figura 4.



Figura 4. Desplazamiento hacia adelante del robot 


\subsection{Modelo cinemático inverso}

A partir del modelo cinemático (ecuación (8)) inverso es posible conocer a qué velocidad angular y el sentido de giro que debe tener cada una de las ruedas con respecto a las otras.

$$
\left[\begin{array}{l}
\omega_{1} \\
\omega_{2} \\
\omega_{3}
\end{array}\right]=\frac{1}{r} \cdot\left[\begin{array}{ccc}
\sin \theta_{1} & -\cos \theta_{1} & -L \\
\cos \theta_{2} & \sin \theta_{2} & -L \\
\cos \theta_{3} & \sin \theta_{3} & -L
\end{array}\right]\left[\begin{array}{l}
x \\
y \\
z
\end{array}\right]
$$

Dichos valores se obtiene remplazando el ángulo correspondiente a la inclinación de cada una de las ruedas respecto al eje de coordenadas.

Por lo tanto para hallar la velocidad angular para cada una de las ruedas se remplaza en las ecuaciones (9), (10), (11) cada uno de los resultados obtenidos en la matriz según corresponda y el valor de L, la cual depende del diseño del robot.

$$
\begin{aligned}
& \omega_{1}=\left(x \cdot \sin \theta_{1}+y \cdot \cos \theta_{1}-z \cdot L\right) \cdot \frac{1}{r} \\
& \omega_{2}=\left(x \cdot \cos \theta_{2}+y \cdot \sin \theta_{2}-z \cdot L\right) \cdot \frac{1}{r} \\
& \omega_{3}=\left(x \cdot \cos \theta_{3}+y \cdot \sin \theta_{3}-z \cdot L\right) \cdot \frac{1}{r}
\end{aligned}
$$

En el caso de la ecuaciones (8) del modelo cinemático inverso, la velocidad angular de la rueda 1 se rige por los valores que se encuentran en la primera fila de la matriz $3 \times 3$, donde el valor que toma $x$ corresponde a la primera columna de izquierda a derecha, $y$ a la segunda columna y $z$ a la tercera (ecuación (12)).

$$
\left[\begin{array}{l}
\omega_{1} \\
\omega_{2} \\
\omega_{3}
\end{array}\right]=\frac{1}{r} \cdot\left[\begin{array}{ccc|c}
0 & -1 & -L \\
\sqrt{3} / 2 & 0,5 & -L \\
-\sqrt{3} / 2 & 0,5 & -L & x \\
z
\end{array}\right]
$$

Por lo tanto para realizar el análisis de la velocidad angular se asignan valores a las variables correspondientes a la posición en $\mathrm{x}, \mathrm{y}, \mathrm{z}$.

Se determina la velocidad angular de las ruedas cuando el robot rota sobre sí mismo, asignando los siguientes valores a las variables de posi- ción y se remplaza en las ecuaciones (9), (10), (11).

$$
\begin{array}{ll|l}
\mathrm{x} & =0[\mathrm{~m}] & \omega_{1}=1,456 \\
\mathrm{y} & =0[\mathrm{~m}] & \omega_{2}=0 \\
\mathrm{z} & =-1[\mathrm{~m}] & \omega_{3}=-1
\end{array}
$$

Como se observa en los resultados, si remplazamos los componentes de la posición del robot se obtiene la velocidad angular para cada una de las ruedas cuando el robot gira sobre sí mismo.

Ahora si se desea conocer la velocidad angular de las ruedas involucradas en el desplazamiento del robot hacia delante solo se remplaza el valor de $\mathrm{x}, \mathrm{y}, \mathrm{z}$ en la ecuación de las Ruedas2 y 3, ya que la Rueda1 se desplazara sobre los rodillos que posee. Tampoco se incluye el valor de $\mathrm{z}$, debido a que el robot no va girar sobre sí mismo.

$$
\begin{aligned}
& \begin{array}{l|l}
\mathrm{x}=1[\mathrm{~m}] & \omega_{1}=0
\end{array} \\
& \mathrm{y}=0[\mathrm{~m}] \quad \omega_{2}=7,21[\mathrm{~m}] \\
& \mathrm{z}=0[\mathrm{~m}] \quad \omega_{3}=-7,21[\mathrm{~m}]
\end{aligned}
$$

Como se ve en los resultados para que el robot se desplazase hacia delante, se requiere que la magnitud de la velocidad angular sea la misma para las Ruedas 2 y 3.

Sin embargo para conseguir que el robot realice el desplazamiento deseado, el modelo matemático del mismo debe apoyarse en la lectura de los encoders, para lograr un sistema realimentado.

\section{ESTUDIO DE LA RESISTENCIA DE MATERIALES}

Calcular la resistencia del material que se quiere utilizar en la construcción del prototipo, permite conocer si dicho material es capaz de resistir las fuerzas que actúan sobre este, mientras realiza las tareas para las cuales fue creado. Por lo tanto, con este estudio se pude determinar el material apto que garantiza una mayor vida útil del prototipo. 
Para realizar dichos cálculos es necesario tener claro cómo va a ser el diseño de la estructura del prototipo, ya que se requiere conocer el área de cada una de las piezas que la conforman. Para este caso particular, las áreas que dé deben calcular son la de un hexágono, el cual corresponde a las bases de la estructura y la de un circulo, que corresponde a las varillas unidas a las bases del robot, como se ve en la figura 5 .

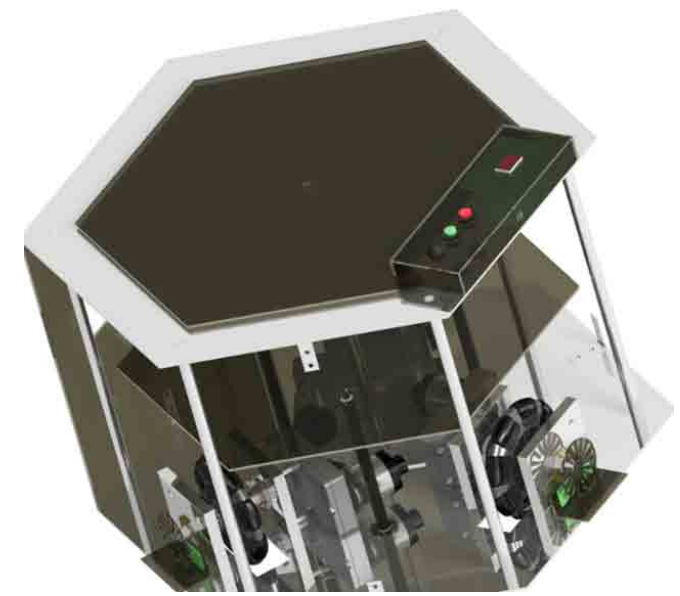

Figura 5. Diseño del prototipo mecánico del robot en CATIA

Por lo tanto, así mismo se debe calcular el esfuerzo que debe soportar cada una de las piezas de la estructura, a partir de las sumatoria de fuerzas que actúan sobre el robot, en este caso es el peso de todos los componentes que lo conforman como motores, baterías, el circuito impreso, etc., Incluso se debe incluir en los cálculos el peso propio de las piezas de la estructura.

La relación a partir de la cual se obtiene el esfuerzo de cualquier material se obtiene a partir de la ecuación (13).

$$
\sigma=\frac{F_{T}}{A}
$$

Donde $\mathrm{F}_{\mathrm{T}}$ es la fuerza total en Newton, $A$ es el área el metros cuadrados y $\sigma$ es el esfuerzo del material, dado en pascales, es decir $\mathrm{N} / \mathrm{m}^{2}$ [7].

A continuación se encuentra el desarrollo de los cálculos realizados para el diseño propuesto del robot, en el cual se contempló el aluminio y acrílico para su construcción.

\section{1. Área del hexágono en aluminio}

Como se muestra en la figura 6, para hallar el área de un hexágono, es necesario hallar a, el cual se denomina apotema, utilizando el teorema de Pitágoras, ya que a junto con I e I/2 forman un triángulo rectángulo (figura 6).



Figura 6. Área del hexágono

Los valores de I e I/2 corresponden al diseño previamente realizado del prototipo, para este caso $\mathrm{I}=0,3[\mathrm{~m}], \mathrm{I} / 2=0,15[\mathrm{~m}]$.

Para hallar la apotema, el perímetro y el área del hexágono se aplican las ecuaciones (14), (15) y (16) respectivamente.

$$
\begin{aligned}
& a=\sqrt{I-\left(\frac{I}{2}\right)^{2}} \\
& a=0,2598[\mathrm{~m}]
\end{aligned}
$$

$$
\begin{aligned}
& \text { Perimetro }=6 \cdot I \\
& \text { Perimetro }=1,8[\mathrm{~m}]
\end{aligned}
$$

$$
\begin{aligned}
& A=\frac{\text { Perimetro } \cdot a}{2} \\
& A=0,23382\left[\mathrm{~m}^{2}\right]
\end{aligned}
$$




\subsection{Peso de la base hexagonal en aluminio (con espesor de $3 \mathrm{~mm}$ )}

Para hallar el peso en Newtons se realiza el producto entre la masa y gravedad (ecuación (19)). La masa de la pieza (ecuación (17) y (18)) esta en función del área (A), el espesor y la densidad del material [8].

Masa $=A \cdot$ Espesor $\cdot$ Densidad del material

Masa $=A \cdot$ Espesor $\cdot$ Densidad del alu min io

$$
\begin{aligned}
& W=m g \\
& m=3,379[\mathrm{~kg}] \\
& g=9,80\left[\mathrm{~m} / \mathrm{s}^{2}\right] \\
& W=33,119[\mathrm{~N}]
\end{aligned}
$$

\section{3. Área del hexágono inferior en acrílico}

$$
\begin{gathered}
\mathrm{I}=0,240[\mathrm{~m}] \\
\mathrm{I} / 2=0,120[\mathrm{~m}]
\end{gathered}
$$

Para hallar la apotema, el perímetro y el área del hexágono se aplican las ecuaciones (14), (15) y (16) respectivamente.

$$
\begin{gathered}
a=0,2087[\mathrm{~m}] \\
\text { Perímetro }=1,44[\mathrm{~m}] \\
A=0,1502[\mathrm{~m}]
\end{gathered}
$$

\subsection{Peso de la base hexagonal en acrílico inferior (con espesor de $3 \mathrm{~mm}$ )}

$$
\begin{gathered}
M(\text { Masa })=0,536[\mathrm{~kg}] \\
W(\text { Peso })=5,257[\mathrm{~N}]
\end{gathered}
$$

\section{5. Área del hexágono superior en acrílico}

Para hallar el área de la base superior en acrílico se debe restar el área rectangular (ecuación (20)). La ecuación (21) corresponde a la interfaz de usuario ubicada en la parte de encima del robot (figura (7)).

$$
\begin{gathered}
A=\text { Area }_{\text {hexagono }}-\text { Area }_{\text {Interfaz usuario }} \\
\text { Area }_{\text {Interfaz usuario }}=a \cdot b
\end{gathered}
$$

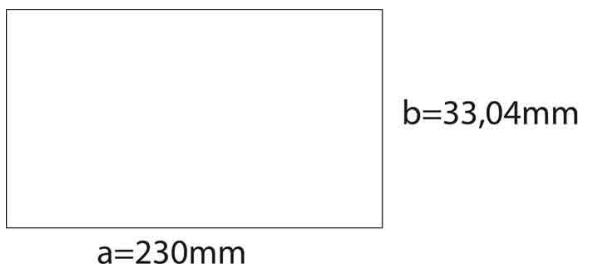

Figura 7. Medidas de los lados de la interfaz de usuario

$$
A=0,1432\left[m^{2}\right]
$$

\subsection{Peso de la base hexagonal en acrílico superior (con espesor de $3 \mathrm{~mm}$ )}

$$
\begin{gathered}
M(\text { Masa })=0,511[\mathrm{~kg}] \\
W(\text { Peso })=5[\mathrm{~N}]
\end{gathered}
$$

Tras haber hallado el peso de las partes que conforman la estructura del prototipo, en la tabla 1 se encuentra relacionada la masa y el peso de las piezas y dispositivos que hacen parte del robot.

Tabla 1. Masas y pesos de los elementos que conforman

\begin{tabular}{|l|c|c|}
\hline \multicolumn{1}{|c}{ Elemento } & $\begin{array}{c}\text { Masa en } \\
\text { Kilogramos }\end{array}$ & $\begin{array}{c}\text { Peso en } \\
\text { Newton }\end{array}$ \\
\hline Batería & $2,30 \mathrm{~kg} \mathrm{c} / \mathrm{u}$ & $22,54 \mathrm{~N}$ \\
\hline Motor & $1,5 \mathrm{~kg} \mathrm{c} / \mathrm{u}$ & $14,7 \mathrm{~N}$ \\
\hline Circuito impreso & $1,5 \mathrm{~kg}$ & $14,7 \mathrm{~N}$ \\
\hline Varillas de aluminio & $927 \mathrm{gr} \mathrm{c} / \mathrm{u}$ & $9,08 \mathrm{~N}$ \\
\hline Carga máxima & $10 \mathrm{~kg}$ & $98 \mathrm{~N}$ \\
\hline $\begin{array}{l}\text { Base hexagonal } \\
\text { inferior en aluminio }\end{array}$ & $3,379 \mathrm{~kg}$ & $33.119 \mathrm{~N}$ \\
\hline $\begin{array}{l}\text { Base hexagonal } \\
\text { superior en aluminio }\end{array}$ & $3,379 \mathrm{~kg}$ & $33,119 \mathrm{~N}$ \\
\hline $\begin{array}{l}\text { Base hexagonal } \\
\text { inferior en acrílico }\end{array}$ & $0,536 \mathrm{~kg}$ & $5,257 \mathrm{~N}$ \\
\hline $\begin{array}{l}\text { Base hexagonal } \\
\text { superior en acrílico }\end{array}$ & $0.511 \mathrm{~kg}$ & 5 \\
\hline
\end{tabular}

\subsection{Esfuerzo de base hexagonal inferior en aluminio}

Para determinar el esfuerzo que soportan las piezas de la estructura, utilizamos los valores del peso en Newtons (tabla 1), los cuales deben sumarse para determinar la fuerza total sobre la pieza, teniendo en cuenta en la sumatoria 
que ítems que hacen parte del robot, afectan o ejercen alguna fuerza sobre la pieza para la cual se está calculando el esfuerzo y se aplica la ecuación (22).

Luego, para saber si el resultado sobrepasa o no el esfuerzo como que posee como tal el material que se desea emplear, se compara el resultado obtenido con la ecuación (22). Por lo tanto si el esfuerzo propio del material es menor que el esfuerzo que debería soportar la pieza, entonces se concluye que el material no es apto para ser utilizado en el proyecto, ya que el peso podría deformar la pieza. Pero si por el contrario el esfuerzo del material es mayor al de la pieza, entonces el prototipo se puede construir con dicho material.

$$
\begin{gathered}
F=\sigma \cdot A \\
\sigma_{\text {aluminio }}=95[\mathrm{Mpa}] \\
F=22,2129 \times 10^{6}[\mathrm{~N}]
\end{gathered}
$$

\subsection{Esfuerzo de base hexagonal superior en aluminio}

$$
\begin{gathered}
\sigma=582,152[\mathrm{~Pa}] \\
\sigma_{\text {aluminio }}=95[\mathrm{Mpa}] \\
F=22,2129 \times 10^{6}[\mathrm{~N}]
\end{gathered}
$$

3.8.1. Esfuerzo de base hexagonal inferior en acrílico

$$
\begin{gathered}
\sigma=193,322[\mathrm{~Pa}] \\
\sigma_{a c r i ́ l i c o}=80[\mathrm{Mpa}] \\
F=12,016 \times 10^{6}[\mathrm{~N}]
\end{gathered}
$$

\subsection{Esfuerzo de base hexagonal superior en acrílico}

$$
\begin{gathered}
\sigma=684,35[\mathrm{~Pa}] \\
\sigma_{\text {acrílico }}=80[\mathrm{Mpa}] \\
F=12,016 \times 10^{6}[\mathrm{~N}]
\end{gathered}
$$

\subsection{Esfuerzo de una varilla de aluminio}

En el caso de las varillas de aluminio, lo que se quiere conocer es el radio de la varilla que soporta la fuerza total de los componentes del robot ecuación (23).

$$
\begin{aligned}
& A=2 \pi \cdot r^{2} \\
& \sigma=\frac{F_{T}}{2 \pi \cdot r^{2}} \\
& r=\sqrt{\frac{F_{T}}{2 \pi \cdot \sigma}}
\end{aligned}
$$

El esfuerzo para una varilla de $1 / 2$ pulgada con radio 12,7 [mm] es de 651,879 [Kpa].

\section{APLICACIÓN DE LA SEGUNDA LEY DE NEWTON}

Con el fin de conocer la fuerza necesaria que deben tener los motores para vencer la fuerza de fricción estática y dinámica que permita al robot desplazarse, se aplica la segunda Ley de Newton (ecuación (24)) [4].

$$
F=m \cdot a
$$

Donde $F$ es la fuerza, $m$ es la masa y $a$ es la aceleración. Para identificar las fuerzas que actuando sobre el robot, se realiza un diagrama de fuerzas como se muestra en la figura 8.



Figura 8. Diagrama de fuerzas del robot

Como se observa en la figura 8 , sobre el robot actúa la fuerza de gravedad $W$ y la fuerza normal $N$. Estas dos fuerzas se anulan, debido a que para una superficie plana poseen la misma magnitud pero diferente dirección, lo cual indica que no hay componente de fuerza en el eje y. Por otra parte, sobre el robot actúan la fuerza de fricción $f$ y la fuerza $F$ que se le imprime al robot para que se desplace, en este caso si hay componente de fuerza en el eje $x$, ya que aun- 
que dichas fuerzas poseen direcciones contrarias, estas cuentan con diferente magnitud. Por lo tanto para hallar la fuerza mínima que deben tener los motores para mover el robot, es necesario hallar la componente de la fuerza en el eje $\mathrm{x}$, a partir de las ecuaciones (25) y (26) [8].

$$
\begin{aligned}
& \sum F_{x}=F+(-f) \\
& \sum F_{x}=m \cdot a_{x}
\end{aligned}
$$

A partir de la ecuación (25) se puede encontrar F (ecuación (26))

$$
F=m \cdot a_{x}+f
$$

Sin embargo antes de aplicar las ecuaciones, se debe obtener la aceleración (ecuaciones (27)), la velocidad en x (ecuación (28)), la fuerza de fricción estática (ecuación (29)) y la dinámica (ecuación (30)) [8].

$$
\begin{gathered}
a_{x}=\frac{V_{x}-V_{0 x}}{t} \\
V_{x}=r \cdot \omega_{m} \\
f_{e}=\mu_{e} \cdot N \\
f_{d}=\mu_{d} \cdot N
\end{gathered}
$$

Donde $V x$ es la velocidad en el eje $x$, Vox es la velocidad en el origen, $r$ es el radio de la rueda, $W m$ es la velocidad angular de la rueda en radianes/segundo, $f_{e}$ y $f_{d}$ es la fuerza de fricción estática y dinámica respectivamente, $\mu_{e} \mathrm{y} \mu_{d}$ son los coeficientes de fricción estáticos y dinámicos [9].

Aunque no es conocido el valor de la velocidad angular en radianes, si lo es en rpm, valor que fue obtenido experimentalmente, contando cuantas revoluciones por minuto completo el eje del motor, cuyo resultado fue $27 \mathrm{rpm}$. Por lo tanto para realizar los cálculos, primero se debe pasar los $27 \mathrm{rpm}$ dados como fm a radianes/segundo remplazando en la ecuación (31).

$$
\begin{aligned}
& \omega_{m}=\frac{f_{m} \cdot 2 \pi}{60} \\
& \omega_{m}=2,827\left[\frac{\mathrm{rad}}{\mathrm{s}}\right]
\end{aligned}
$$

Tras obtener el valor de Wm, se halla el valor de la velocidad lineal y de la aceleración.

$$
\begin{gathered}
r=0,12[\mathrm{~m}] \\
V_{x}=0,169[\mathrm{~m} / \mathrm{s}] \\
a_{x}=0,169\left[\mathrm{~m} / \mathrm{s}^{2}\right]
\end{gathered}
$$

Con el valor de la aceleración, se debe obtener los valores de la fuerza de fricción estática y dinámica, para eso es necesario hallar la fuerza normal (ecuación (32)).

$\mathrm{m}=39.494$ [kg] (Resultado obtenido de la sumatoria de las masas de la tabla 1).

$$
\begin{aligned}
& N-W=0 \\
& N=W \\
& N=m \cdot g \\
& N=378,04[N]
\end{aligned}
$$

Finalmente, se aplica la ecuación (26) para encontrar la componente de la fuerza en x para una fuerza de fricción estática y una dinámica [9].

$$
\begin{aligned}
& f_{e}=200,19[\mathrm{~N}] \\
& f_{d}=122,78[\mathrm{~N}]
\end{aligned}
$$

Por lo tanto, la fuerza que deben ejercen los motores para superar la fuerza de fricción cuando el robot esta en reposo y empieza a desplazarse es igual a 200,19 N. Por otro lado, en consecuencia, los motores deben tener una fuerza de torque mínima de $122.78 \mathrm{~N}$ para que el robot continúe desplazándose [10] [11].

\section{METODOLOGÍA}

El objetivo del diseño y construcción de este prototipo es que sea capaz de llevar objetos a tres diferentes rutas, clasificándolos según el peso en un rango de 1 a $10 \mathrm{~kg}$ máximo. Para lograr que el robot cumpla su tarea se imple- 
mento un sensor de fuerza [12], encargado de medir el peso del objeto (partiendo de la premisa que el peso es una fuerza), encoders y un sensor de proximidad [13] para la detección de obstáculos. En la figura 9 se muestra el diagrama de bloques del control general que se implemento.

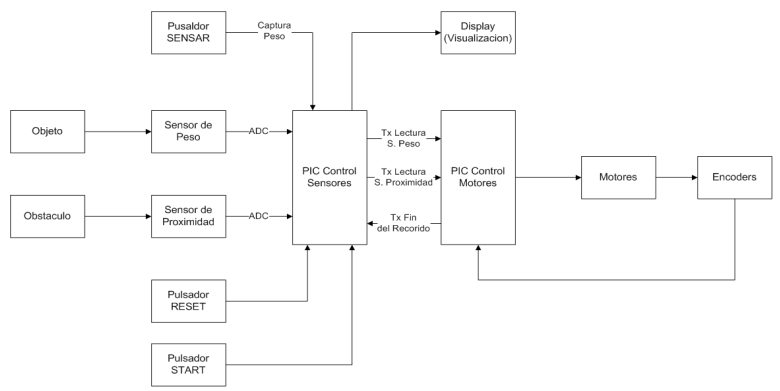

Figura 9. Diagrama de bloques control general

En la figura 9 las entradas del sistema llegan directamente el PIC encargado de controlar los sensores, el cual constantemente verifica los cambios que se puedan producir en las entradas. Por lo tanto cuando se produce un cambio el PIC lo visualiza y le informa al PIC encargado del control de los motores, los cuales pueden representar la selección de la ruta basada en el peso del objeto o la detección de un obstáculo.

Por otra parte el control de los motores es en lazo cerrado [14], ya que por medio de los encoders se realiza la retroalimentación del sistema, indicando con el número de vueltas, cuanta distancia ha avanzado el robot. Internamente el microcontrolador usa la información obtenida de los encoders para regular el PWM [15] y por ende la velocidad de los motores.

El algoritmo del PIC Control Motores posee tres variables que son ruta, encoder y sensor de proximidad, una vez el PIC recibe el código de la ruta, pasa a la selección de la misma dentro de las tres opciones con las que cuenta, luego realiza el cálculo del PWM según el ruta escogida y comienza a recorrer el vector que contiene los movimientos que debe realizar el robot, como ir hacia adelante, girar hacia la derecha o izquierda según corresponda. Mientras el vector sigue ejecutándose, entonces también se ejecutara la rutina del PWM, la cual saca un tren de pulsos por uno de los puertos del PIC y va a la etapa de potencia del robot haciendo que se muevan los motores. En este punto los encoders empiezan a recoger información del número de vueltas que ha dado el eje del motor y la envía al PIC cerrando el lazo de control, como se muestra en la figura 10.

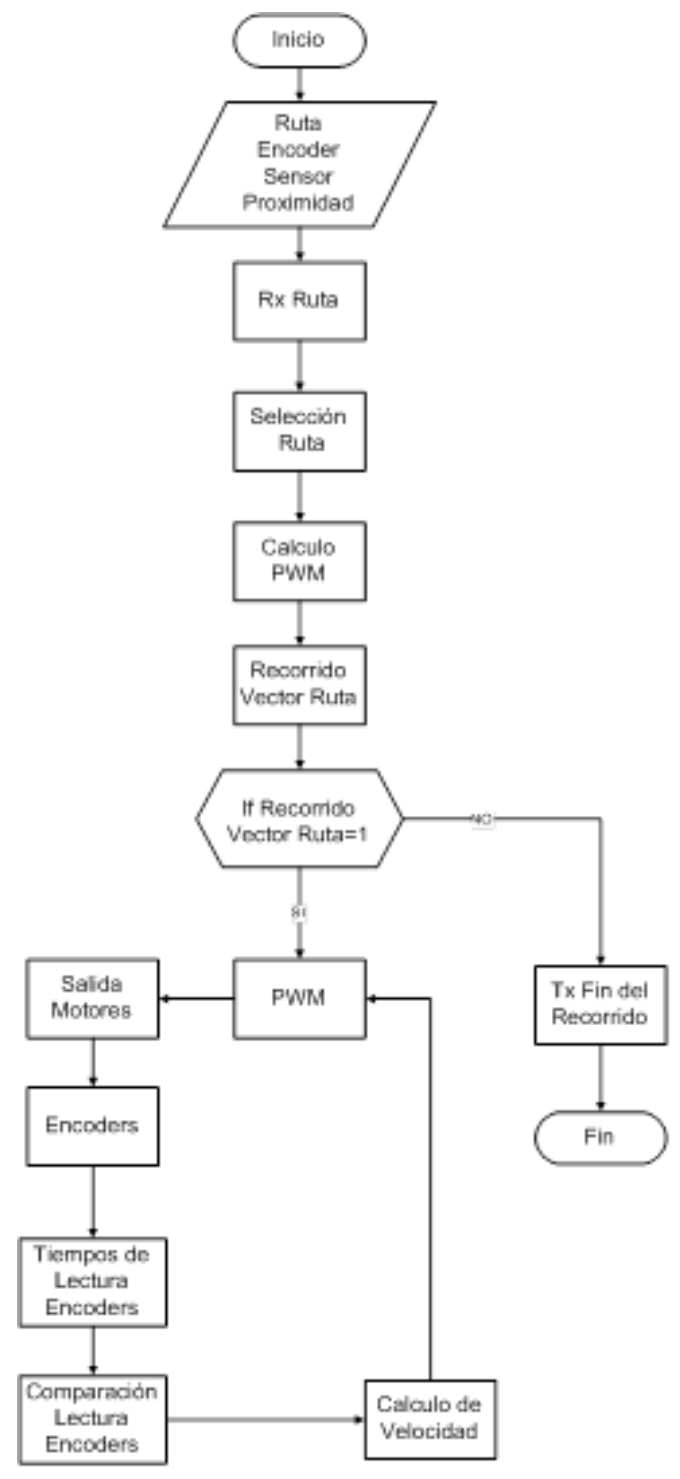

Figura 10. Diagrama de flujo PIC control motores

\section{RESULTADOS}

En las figuras 11, 12 y 13 se muestran la evolución en la construcción del prototipo.

En figura 11 se observa la realización de prue- 
bas mecánicas al prototipo, conectando únicamente los motores a la batería con el fin de ver como se esta desplazando, es decir si lo hace en línea recta o tiende hacer una curva, lo cual se puede deber a que los componentes del robot no están simétricamente colocados, la carga no esta colocada en el centro del robot, lo cual es muy importante para conservar el centro de gravedad del mismo o que una rueda va a mayor velocidad que la otra.

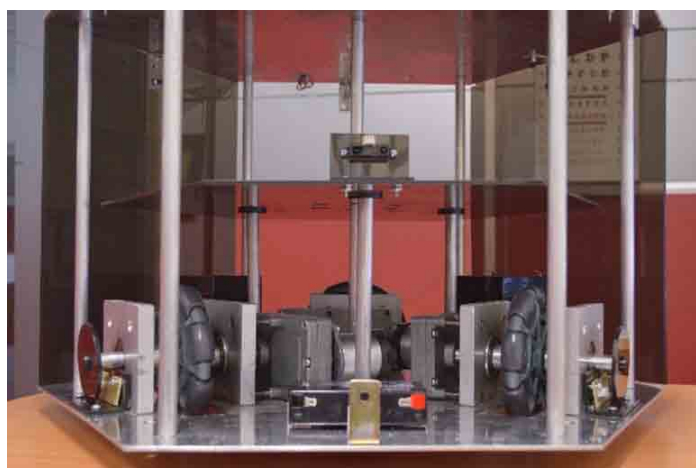

Figura 11. Estructura en aluminio.

Pruebas de desplazamiento

En la figura 12 se realizan pruebas de movimiento con el control electrónico incorporado, con el fin de determinar si el control y el algoritmo implementado funcionan correctamente o requiere cambios.

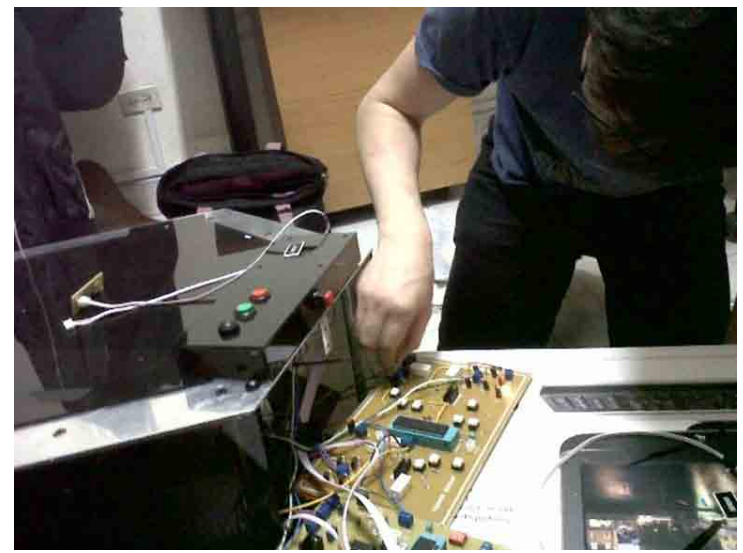

Figura 12. Pruebas de desplazamiento con control electrónico.

En la figura 13 se aprecia como se ve el robot ensamblado totalmente. El robot cuenta con un panel de control conformado por el botón de
Start, Reset, On-Off y un display doble de siete segmentos.

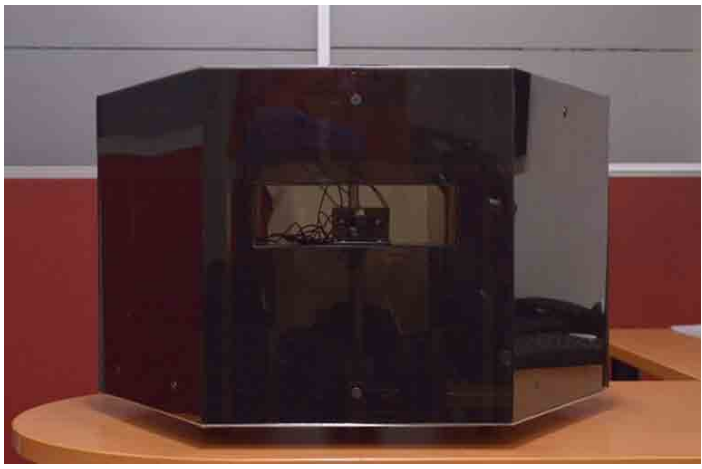

Figura 13. Vista del prototipo terminado

\section{CONCLUSIONES}

Para la elaboración fue vital entender a fondo el funcionamiento del sistema de locomoción de un robot omnidireccional teniendo un modelo matemático que lo soporte, ya que esto permite predecir el desplazamiento del robot en un sistema de coordenadas, así como también permite determinar la velocidad angular con la que deben trabajar los motores, para lograr que el robot de desplace en la forma deseada, lo cual a la hora de programar el prototipo facilita dicho proceso, ya que las ecuaciones y los resultados obtenidos del modelo cinemático se pueden incorporar dentro del programa como un algoritmo, haciendo más eficaz el control sobretodo de los motores, ya que es posible conocer cuanto se debe desplazar el robot y a que velocidad para lograr su objetivo.

Por otra parte, se puede inferir que el aluminio es un material óptimo para la construcción del chasis del robot, ya que por ejemplo en el caso de la base hexagonal inferior de aluminio, la cual debe resistir sin deformarse prácticamente todo el peso en si del robot, puede llegar soportar hasta $62000 \mathrm{~N}$ más de lo que le exige el diseño. Esto debido a que el esfuerzo que debe resistir de acuerdo con el diseño mecánico, es apenas de 1537.4Pa en comparación a 95MPa que puede llegar a resistir. Por lo tanto se trata de un material muy resistente a pesar de ser liviano. De igual manera ocurre con la base en acrílico, la cual también es bastante resistente y 
soporta perfectamente el peso de los elementos que provocan una fuerza sobre la misma. Por otro lado para el caso de las varillas, como se puede observar en los resultamos, cada varilla puede medir aproximadamente al menos $1 \mathrm{~mm}$ de diámetro para lograr soportar la estructura del robot, es decir que cada varilla de media pulgada utilizada en el prototipo solo requiere un esfuerzo mínimo de $642 \mathrm{KPa}$, cuando el esfuerzo del material llega hasta $95 \mathrm{MPa}$, lo que indica que las varillas seleccionadas pueden resistir aun más peso y por lo tanto son más que optimas para la construcción de la estructura del prototipo.

Por otra parte al aplicar la Segunda Ley de Newton, se logro obtener la fuerza que deben tener los motores, lo cual es fundamental ve-

\section{Referencias Bibliográficas}

[1] H. Henderson; Modern Robotics Building Versatiles Machine. Chelsea House Publishers, pp. 15-19, New York, 2006.

[2] J. Craig; Introducción a la Robótica. 3ed., Pearson Education, México, 2006.

[3] Three Engineers, Hundreds of Robots, One Warehouse. IEEE Spectrum [En linea], consultado en Septiembre 17 de 2013 Disponible en: http://spectrum. ieee.org/robotics/robotics-software/ three-engineers-hundreds-of-robots-onewarehouse $/ 0$

[4] J. Fletcher, S. Ho, J. Kloess, R. Raj y S. Vozar; Round a Bot, holonomic Invert Pendunlum Robot. Ann Arbor, 2010.

[5] F. Cerro; "Vehículo compacto omnidireccional con ruedas no convencionales", Tesis de Pregrado. Escuela Técnica Superior de Ingeniería Industrial, Barcelona, 2004.

[6] F. G. Pin, S. M. Killough; A New Family of Omni-Directional and Holonomic Wheeled Platforms for Mobile Robots, IEEE Trans. Rob. Autom., 10_4_, pp. 480-489, 1994.

[7] L. García; Modelo Cinemático y Control de Robots Móviles con ruedas, $\mathrm{PhD}$ Tesis, rificar que los motores que se escogieron son los apropiados para el proyecto o determinar la relación de reducción con el fin de lograr que el torque que proporcione el eje del motor sea el indicado para lograr que el robot se mueva. A raíz de los resultados, se observo que es necesario aplicar una mayor fuerza cuando se desea mover un cuerpo que se encuentra en reposo que uno que ya se encuentra en movimiento.

Finalmente de las pruebas realizadas al robot se puede concluir que al tratarse de un prototipo cuya forma es totalmente simétrica, la carga se debe colocar en el centro del robot para conservar el centro de masa, de lo contrario el robot tiende a frenarse o cambia la dirección de su desplazamiento.
[8] J. Gere; Mecánica de Materiales, Thomson, 6 ed., México, 2006.

[9] F. Beer, R. Johnston, J. Dewolf; Mecánica de Materiales, McGraw Hill, 3 ed., México D.F, 2001.

[10] R. Serway; Física para Ciencias e Ingenierías. Thomson, 6 ed., Vol. I., México, 2005.

[11] Y. Freedman, S. Zemansky; Física Universitaria. Pearson Education, 12 ed., Vol. I., México, 2009.

[12] R. Palàs-Areny, J. G. Webster; Sensors and Signal Conditioning, John Wiley \& Sons, 2 ed., United States of America, 2001.

[13] D. S. Albán, F. R. Salazar; Diseño e Implementación de Sensores de Temperatura, Presión y Proximidad en un Prototipo de Piel Sensible para Robots Compañeros, Tesis de Pregrado, Escuela Politécnica del Ejercito sede Latacunga, Latacunga, 2011.

[14] K. Ogata, Ingeniería de Control, Pearson Educación, 3ed., México, 1998.

[15] M. H. Rashid, Electrónica de Potencia, Prentice Hall, 2 ed., México, 1995. 